study in 5- and 6-year-old children. Dev Med Child Neurol Nov 2002;44:753-760). (Marielle Kroes MD PhD, University Hospital of Maastricht, Department of Neurology, PO Box 5800, 6202 AZ, Maastricht, the Netherlands).

COMMENT. The significance of motor performance as an indicator of attentional problems and ADHD has been stressed by previous investigators. Denckla $(1973,1974)$ and Denckla and colleagues (1985) used speed of motor function in repetitive tapping movements of finger, hand, and foot to assess motor performance in normal children and children with dyslexia with and without attention problems. Children with ADHD were significantly slower in motor function than controls.

Huttenlocher et al, (1990) found that "soft" neurologic signs were of predictive value for learning disabilities in preschool children, aged 3 to 5 years. A poor neurologic test score at age 5 years correlated with a lower Full-Scale IQ at age 7, and neurologic soft signs accurately identified almost all the children who needed special educational services. Soft neurologic signs and so-called "minimal brain dysfunction (MBD)" have previously been correlated with symptoms of ADHD and a beneficial response to stimulant medication (Millichap, 1974; see Attention Deficit Hyperactivity and Learning Disorders (PNB Publishers, 1999).

The present investigators evaluated both quantitative and qualitative aspects of motor performance, using the Maastricht Motor Test (MMT). The MMT includes 70 items organized in 4 domains: static balance (stork position), dynamic balance (hopping), ball skills (number of catches), and diadochokinesia and manual dexterity (speed of tapping the hand). Qualitative aspects of motor performance were predictive of ADHD in 5- to 6-yearold children.

Early motor skills and language development in at-risk dyslexic children. In a Finnish study of 88 children with familial risk for dyslexia cf 88 without risk, children with familial risk for dyslexia and slow motor development had a smaller vocabulary and spoke in shorter phrases at 18 and 24 months than other children. (Viholainen H, Ahonen T, Cantell M et al. Dev Med Child Neurol Nov 2002;44:761-769).

\title{
ADHD COMORBIDITY, BEHAVIOR, AND TIC SEVERITY
}

The association of disruptive behavior with social, adaptive, and family functioning in Tourette syndrome (TS), with and without comorbid attention-deficit/hyperactivity disorder (ADHD), was evaluated in 207 children (144 boys and 63 girls) between the ages of 7 and 18 years, in a study at Yale University School of Medicine, New Haven, CT. Children with TS-only $(\mathrm{n}=42)$ did not differ from unaffected controls (61) on the parent ratings of aggression and delinquent behavior or on the teacher ratings of conduct disorders. Children with TS+ADHD $(n=52)$ had significantly greater ratings of disruptive behavior than controls and similar to children with ADHD only $(n=52)$. Aggression and delinquency contributed to impaired social and family functioning. Comorbid ADHD is highly associated with disruptive behavior and functional impairment in children with TS. Disruptive behavior adds to the burden of social and family dysfunction. (Sukhodolsky DG, Scahill L, Zhang H et al. Disruptive behavior in children with Tourette's syndrome: association with ADHD comorbidity, tic severity, and functional impairment. J Am Acad Child Adolesc Psychiatry January 2003;42:98-105). (Reprints: Dr Scahill, Yale University 
School of Medicine, Child Study Center, 230 South Frontage Road, New Haven, CT 06520).

COMMENT. Children and adolescents with uncomplicated Tourette syndrome (TS) are not at risk for aggressive and disruptive behavior, whereas those with comorbid TS +ADHD have a significantly higher prevalence of behavioral and conduct disorders, similar to that found with ADHD alone. Aggressive and delinquent behaviors have a negative impact on social and family functioning, adding further burdens to the quality of life of children with comorbid TS/ADHD.

\section{PROGNOSIS OF LANGUAGE-DELAYED AND AUTISTIC PRESCHOOL CHILDREN}

The outcome of 18 children (13 males, 5 females) with severe developmental language delay and some features of autism (not fulfilling all criteria), examined at preschool age (mean age 4 years 4 months) and again 4 years later (mean age 8 years 7 months), was evaluated at the Institute of Child Health, London, UK. At follow-up, 5 children had continuing language disorder and fulfilled diagnostic criteria for childhood autism, 4 had atypical autism with associated language disorder, and 9 had atypical autism with improved language skills. Degree of social communication impairments and repetitive behaviors at preschool evaluation correlated with severity of autism symptoms at follow-up. Parents' concerns initially were mainly about speech and language whereas at follow-up, social difficulties and social isolation were the primary concerns and inability to relate to peers. The presence of restricted, repetitive, and stereotyped behaviors in preschoolers with language delay is a negative prognostic indicator of a diagnosis of autism in later childhood. (Michelotti J, Charman T, Slonims V, Baird G. Follow-up of children with language delay and features of autism from preschool years to middle childhood. Dev Med Child Neurol Nov 2002;44:812-819). (Respond: Dr Tony Charman, Behavioural and Brain Science Unit, Institute of Child Health, 30 Guildford Street, London WC1N 1EH, UK.).

COMMENT. The relation between language delay and autism presents a diagnostic challenge in preschool children. Impairments of social interaction and repetitive behaviors reported by parents or preschool care-givers are considered risk factors for a diagnosis of autism in later childhood, especially in association with language delay. An early diagnosis of autistic spectrum disorder will permit access to appropriate interventional services.

\section{EFFECT OF LEVETIRACETAM ON MOTOR LEARNING}

The effect of the antiepileptic drug, levetiracetam, on rapid motor learning was tested in 10 healthy, right-handed volunteers (age, 22 to 43 years) by investigators from the National Institutes of Health, Bethesda, MD, and Yonsei University, Seoul, Korea. Maximum pinch force of the left thumb and index finger was determined by a pinch gauge, and the average pinch force of 5 trials was calculated before and after 30 minutes of practice at $0.5 \mathrm{~Hz}$. Either $3000 \mathrm{mg}$ of levetiracetam or placebo was administered 1 hour before the experiment. With placebo, pinch practice significantly increased pinch 